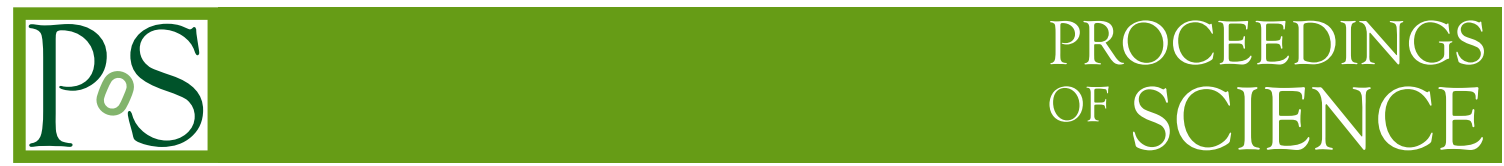

\title{
Charmonium and charmonium-like states with BABAR
}

\author{
Elisa Guido*i \\ INFN - Sezione di Genova, Via Dodecaneso 33 I-16146 Genova (Italy) \\ E-mail: elisa.guido@ge.infn.it
}

\begin{abstract}
We present a study of the decays $B^{0,+} \rightarrow J / \psi \pi^{+} \pi^{-} \pi^{0} K^{0,+}$, on the BABAR data sample, showing an evidence for the decay $X(3872) \rightarrow J / \psi \omega$. The analysis of the $\pi^{+} \pi^{-} \pi^{0}$ mass distribution from $X(3872)$ favors a negative-parity assignment. We also present a study of the processes $\gamma \gamma \rightarrow$ $K_{S}^{0} K^{ \pm} \pi^{\mp}$ and $\gamma \gamma \rightarrow K^{+} K^{-} \pi^{+} \pi^{-} \pi^{0}$. We observe the $\eta_{c}(1 S), \chi_{c 0}(1 P)$ and $\eta_{c}(2 S)$ resonances produced in two-photon interactions and decaying to $K^{+} K^{-} \pi^{+} \pi^{-} \pi^{0}$. We find evidence for the $\chi_{c 2}(1 P)$ decaying to the same final state. We provide the most precise measurement of mass and width of the $\eta_{c}(2 S)$.
\end{abstract}

The 2011 Europhysics Conference on High Energy Physics, EPS-HEP 2011,

July 21-27, 2011

Grenoble, Rhône-Alpes, France

\footnotetext{
*Speaker.

${ }^{\dagger}$ On behalf of the BABAR Collaboration.
} 


\section{Introduction}

The BABAR experiment - which is described in detail elsewhere $[1,2]$ - has recently obtained several important results in charmonium and charmonium-like physics. Below the $D \bar{D}$ threshold, the expected charmonium states are well established and their properties, when measured, are in good agreement with predictions. Nevertheless, in the last years, a plethora of unexpected states has been observed above the $D \bar{D}$ threshold. This non-regular charmonium genealogy started with the observation of the $X(3872)$ resonance [3], and the interpretation of these states is still unclear.

In the studies of the charmonium(-like) spectrum, the complete data sample collected at the PEP-II asymmetric energy $e^{+} e^{-}$collider has been used. This sample consists of integrated luminosities of $425.6 \mathrm{fb}^{-1}$ recorded at an energy in the $e^{+} e^{-}$center-of-mass (CM) frame equal to the $\Upsilon(4 S)$ mass, $28.0 \mathrm{fb}^{-1}$ at the $\Upsilon(3 S)$, and $13.6 \mathrm{fb}^{-1}$ at the $\Upsilon(2 S)$. Samples collected at CM energy 30-40 MeV below each $\Upsilon$ peak, which correspond to $\sim 10 \%$ of the total luminosity are used as well.

Here we present two recent results obtained by $B A B A R$. The first concerns the evidence for the decay $X(3872) \rightarrow J / \psi \omega$ reported in the study of the $B^{0,+} \rightarrow J / \psi \pi^{+} \pi^{-} \pi^{0} K^{0,+}$ processes [4], while the second involves the study of the charmonium resonances produced in two-photon interactions, resulting in the $K_{S}^{0} K^{ \pm} \pi^{\mp}$ and $K^{+} K^{-} \pi^{+} \pi^{-} \pi^{0}$ final states [5].

\section{Evidence for the decay $X(3872) \rightarrow J / \psi \omega$}

The $X(3872)$ state has been primarily observed in decays to $J / \psi \pi^{+} \pi^{-}$[3]. Evidence for $X(3872) \rightarrow J / \psi \gamma$ decay has established positive C-parity for the resonance [6]. Moreover, analyses of the $\pi^{+} \pi^{-}$mass distribution and of the angular distribution have restricted the available $J^{P}$ values for $X(3872)$ to $1^{+}$or $2^{-}$[7].

Here we analyze the decays $B^{0,+} \rightarrow J / \psi \pi^{+} \pi^{-} \pi^{0} K^{0,+}$, selecting the mass window for the three-pion $(3 \pi)$ system in the $\omega$ region as $0.740<m_{3 \pi}<0.795 \mathrm{GeV} / \mathrm{c}^{2}$. The same processes have already been analyzed in [8], but selecting $0.770<m_{3 \pi}<0.795 \mathrm{GeV} / \mathrm{c}^{2}$, with the aim of confirming the $Y(3490) \rightarrow J / \psi \omega$ decays. Apart from the enlarged $\omega$ mass window for the $3 \pi$ system, the same selection criteria as in [8] are applied.

We look at the $J / \psi \omega$ mass distribution on data collected at the $\Upsilon(4 S)$ energy, corresponding to $\sim 467 \times 10^{6} B \bar{B}$ pairs, for both charged and neutral $B$ decays. The mass spectra are shown in Fig. 1 (a, c), with a zoom in the low-mass region in (b). The fit components are the $X(3872)$ resonance, the $Y(3940)$ resonance, and a non-peaking background contribution. We find evidence for the $X(3872)$ decaying to $J / \psi \omega$ with a $4.0 \sigma$ significance, when considering both statistical and systematic uncertainties. The mass of this resonance is measured to be: $m_{X(3872)}=$ $3873.0_{-1.6}^{+1.8}$ (stat.) \pm 1.3 (syst.) $\mathrm{MeV} / \mathrm{c}^{2}$. We also measure mass and width of the $Y(3940)$ resonance: $m_{Y(3940)}=3919.1_{-3.4}^{+3.8}$ (stat.) \pm 2.0 (syst.) $\mathrm{MeV} / \mathrm{c}^{2}$ and $\Gamma_{Y(3940)}=31_{-8}^{+10}$ (stat.) \pm 5 (syst.) $\mathrm{MeV}$.

In Fig. 1 (d, e, f) the mass distribution for the $3 \pi$ system is shown for charged decays, neutral decays, and combining them, respectively. Monte Carlo (MC) simulated events with either $S$ - or $P$-wave distributions are superimposed on the distribution obtained when combining charged and neutral $B$ decays. The analysis favors the $P$-wave description, so that among the $J^{P}$ values still allowed for the $X(3872)$ meson, $2^{-}$is preferred. For this assignment, the $X(3872)$ mass falls within the broad range available to the $\eta_{c 2}(1 D)$ charmonium state. 

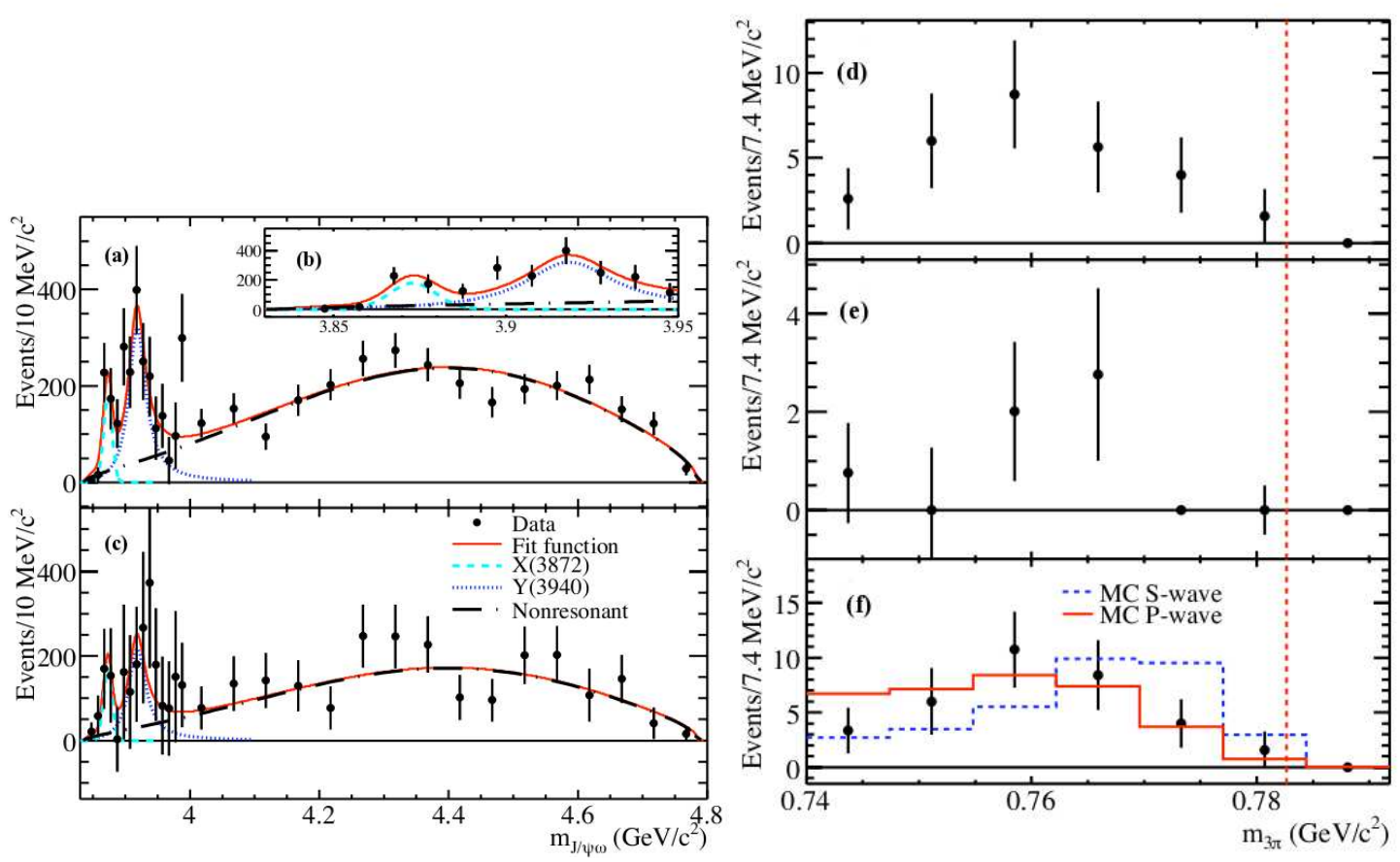

Figure 1: The $m_{J / \psi \omega}$ distribution (a) for $B^{+}$decay, (b) for the low-mass region in detail, and (c) for $B^{0}$ decay. In each case, the curves indicate the fit result. The $m_{3 \pi}$ distribution for events with $3.8625<m_{J / \psi \omega}<3.8825$ $\mathrm{GeV} / \mathrm{c}^{2}$ is shown in (d) for $B^{+}$decay, (e) $B^{0}$ decay, and (f) for the combined distributions. The vertical dashed line represents the nominal $\omega$ mass. In (f), the solid (dashed) histogram represents the $m_{3 \pi}$ distribution for MC $J / \psi \omega P$-wave $(S$-wave) events.

\section{Observation of $\eta_{c}(1 S, 2 S) \rightarrow K^{+} K^{-} \pi^{+} \pi^{-} \pi^{0}$ in two-photon interactions}

The $\eta_{c}(1 S, 2 S)$ are established states in the charmonium spectrum, but many of their properties, especially for the $\eta_{c}(2 S)$, are not established. In the analysis presented here we study the two-photon process $e^{+} e^{-} \rightarrow \gamma \gamma e^{+} e^{-} \rightarrow f e^{+} e^{-}$, where $f$ is either a $K_{S}^{0} K^{ \pm} \pi^{\mp}$ or a $K^{+} K^{-} \pi^{+} \pi^{-} \pi^{0}$ final state. The $e^{+} e^{-}$pair in the final state remains undetected, and the two photons are quasi-real. This implies that the allowed $J^{P C}$ values of the initial state are $0^{ \pm+}, 2^{ \pm+}$, with $J>2$ suppressed by decay phase space. The final state $K_{S}^{0} K^{ \pm} \pi^{\mp}$ cannot have $J^{P}=0^{+}$. The $K_{S}^{0}$ is reconstructed in the $K_{S}^{0} \rightarrow \pi^{+} \pi^{-}$mode, and the $\pi^{0}$ via decay to two photons. All of the available integrated luminosity $\left(519.2 \mathrm{fb}^{-1}\right)$ is used for this study.

Background contributions come from random combinations of particles from $e^{+} e^{-}$annihilation, other two-photon collisions, and processes involving initial state radiation (such as $J / \psi$ production). The signal is identified by the number of charged tracks in the final state, the number of additional reconstructed photons, the transverse momentum distribution and the missing mass of the event. The signals are extracted by means of a binned maximum-likelihood fit to the invariant mass distributions of final state particles, which are shown in Fig. 2. Each fit contains several components: the $\eta_{c}(1 S, 2 S)$, the $\chi_{c 0,2}(1 P)$, and the non-resonant and resonant backgrounds. All the signal yields are corrected for the presence of peaking backgrounds. No signal is found for the $\chi_{c 2}(2 P)$. 
Table 1: Measured $\Gamma_{\gamma \gamma} \times \mathscr{B}$ products for the transitions examined in [5]. $\Gamma_{\gamma \gamma}$ indicates the two-photon coupling. The first error is statistical, the second systematic. The upper limits are at the $90 \%$ confidence level.

\begin{tabular}{l|c} 
Transition & $\Gamma_{\gamma \gamma} \times \mathscr{B}(\mathrm{keV})[5]$ \\
\hline$\eta_{c}(1 S) \rightarrow K \bar{K} \pi$ & $0.386 \pm 0.008 \pm 0.021$ \\
$\chi_{c 2}(1 P) \rightarrow K \bar{K} \pi$ & $(1.8 \pm 0.5 \pm 0.2) \times 10^{-3}$ \\
$\eta_{c}(2 S) \rightarrow K \bar{K} \pi$ & $0.041 \pm 0.004 \pm 0.006$ \\
$\chi_{c 2}(2 P) \rightarrow K \bar{K} \pi$ & $<2.1 \times 10^{-3}$ \\
\hline$\eta_{c}(1 S) \rightarrow K^{+} K^{-} \pi^{+} \pi^{-} \pi^{0}$ & $0.190 \pm 0.006 \pm 0.028$ \\
$\chi_{c 0}(1 P) \rightarrow K^{+} K^{-} \pi^{+} \pi^{-} \pi^{0}$ & $0.026 \pm 0.004 \pm 0.004$ \\
$\chi_{c 2}(1 P) \rightarrow K^{+} K^{-} \pi^{+} \pi^{-} \pi^{0}$ & $(6.5 \pm 0.9 \pm 1.5) \times 10^{-3}$ \\
$\eta_{c}(2 S) \rightarrow K^{+} K^{-} \pi^{+} \pi^{-} \pi^{0}$ & $0.030 \pm 0.006 \pm 0.005$ \\
$\chi_{c 2}(2 P) \rightarrow K^{+} K^{-} \pi^{+} \pi^{-} \pi^{0}$ & $<3.4 \times 10^{-3}$
\end{tabular}
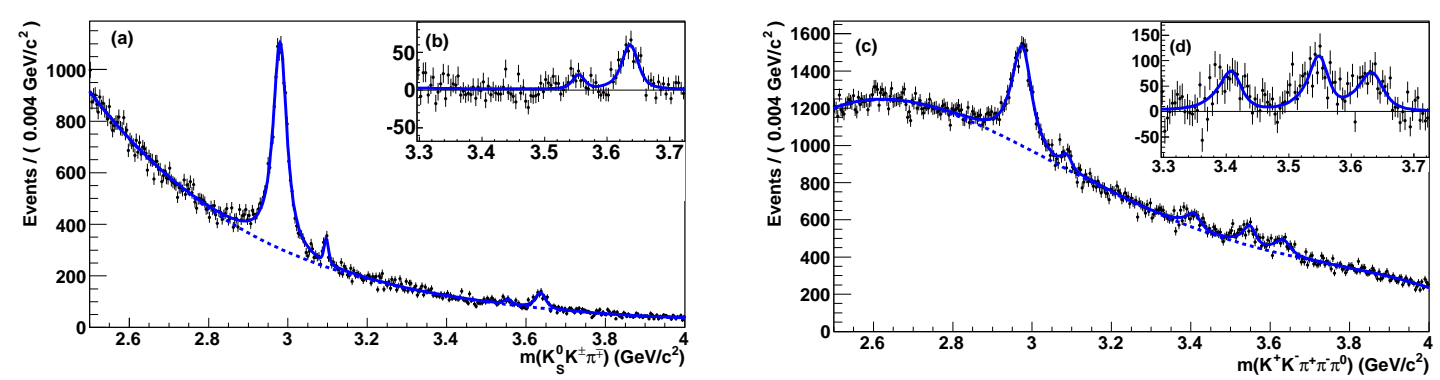

Figure 2: Fit to (a) the $K_{S}^{0} K^{ \pm} \pi^{\mp}$ and (c) the $K^{+} K^{-} \pi^{+} \pi^{-} \pi^{0}$ mass spectrum. The background-subtracted distributions are shown in (b) and (d), respectively. The solid lines show the fit results, the dashed lines the background contributions.

We provide the most precise mass and width measurements for the $\eta_{c}(2 S): M\left(\eta_{c}(2 S)\right)=$ $3638.5 \pm 1.5$ (stat.) \pm 0.8 (syst.) $\mathrm{MeV} / \mathrm{c}^{2}$, and $\Gamma\left(\eta_{c}(2 S)\right)=13.4 \pm 4.6$ (stat.) \pm 3.2 (syst.) MeV. Several branching fractions are also measured, as summarized in Table 1. In particular, we report observation of the $\eta_{c}(2 S)$ exclusive hadronic decay to a final state other than $K \bar{K} \pi$.

\section{References}

[1] B. Aubert et al. (BABAR Collaboration), Nucl. Instrum. Methods Phys. Res. A 479 (2002) 1.

[2] W. Menges, IEEE Nucl. Sci. Symp. Conf. Rec. 5 (2006) 1470.

[3] S. K. Choi et al. (Belle Collaboration), Phys. Rev. Lett. 91 (2003) 262001.

[4] P. del Amo Sanchez et al. (BABAR Collaboration), Phys. Rev. D82 (2010) 011101(R).

[5] P. del Amo Sanchez et al. (BABAR Collaboration), Phys. Rev. D84 (2011) 012004(R).

[6] B. Aubert et al. (BABAR Collaboration), Phys. Rev. Lett. 102 (2009) 132001.

[7] A. Abulencia et al. (CDF Collaboration), Phys. Rev. Lett. 96 (2006) 102002; Phys. Rev. Lett. 98 (2007) 132002.

[8] B. Aubert et al. (BABAR Collaboration), Phys. Rev. Lett. 101 (2008) 082001. 